\title{
Stroke Characteristics of Lifesaving Maximal Board Paddling: A Comparison of Japanese Elite and Sub-elite Paddlers
}

\author{
Motoyoshi Miyama1, Azusa Uematsu², Tatsuya Urata ${ }^{3}$, Hiroya Endo ${ }^{4}$, \\ Hirokazu Arai ${ }^{5}$, Kentaro Nakatsuka ${ }^{6}$ and Masanobu Araki ${ }^{7}$ \\ ${ }^{1}$ Faculty of Management and Information Sciences, Josai International University, 1 Gumyo, Togane, Chiba, 283-8555, Japan \\ Motoyoshi Miyama (miyama@jiu.ac.jp) \\ ${ }^{2}$ Department of Health and Sport Sciences, Premedical Sciences, Dokkyo Medical University, 880 Kitakobayashi, Mibu-machi, \\ Shimotsuga-gun, Tochigi, 321-0293, Japan \\ ${ }^{3}$ Department of social welfare, Kobe University of Welfare, 1966-5 Takaoka, Fukusaki-machi, Kanzaki-gun, Hyogo, \\ 679-2217, Japan \\ ${ }^{4}$ Faculty of Sport Science, Nippon Sport Science University, 7-1-1 Fukasawa, Setagayaku, Tokyo, 158-8508, Japan \\ ${ }^{5}$ Faculty of Health \& Sport Sciences, Ryutsu Keizai University, 120 Hirahata, Ryugasaki, Ibaraki, 301-8555, Japan \\ ${ }^{6}$ Graduate School of Technology, Industrial and Social Sciences, Tokushima University, 1-1 Minamijyosanjima, Tokushima, \\ 770-8502, Japan \\ ${ }^{7}$ Faculty of Sport Sciences, Nihon Fukushi University, 35-6 Aza-Egemae, Ooaza-Okuda, Mihama-cho, Chita-gun, Aichi, \\ 470-3295, Japan \\ [Received April 23, 2018; Accepted October 17, 2018; Published online November 20, 2018]
}

\begin{abstract}
This study compared the differences in board velocity, stroke rate, and stroke length during 40-m maximal board paddling between elite and sub-elite paddlers. Sixteen Japanese male lifesavers (eight each of elite and sub-elite paddlers) performed 40-m maximal board paddling using the knee paddling (K-paddling) and prone paddling (P-paddling) techniques, respectively. Board velocity (BV), stroke rate (SR), and stroke length (SL) were calculated from videorecorded time trials. Board velocity was significantly higher in the elite group with both paddling techniques. The present study identified that the differences in the BVs between the two groups were mainly due to greater SL in K-paddling and higher SR in P-paddling, respectively, in the elite group. Furthermore, peak BVs were significantly related to SL with both paddling techniques in the elite group. Therefore, board paddlers and coaches should understand the differences in the SR-SL ratios for different performance levels based on the results of the present study. They should focus on the increases in the SL and SR values for K-paddling and P-paddling, respectively, based on the SR-SL ratios of the elite group in the present study, when improving sub-elite paddlers' BV to reach elite paddler levels.
\end{abstract}

Keywords: Board velocity, stroke rate, stroke length, knee paddling, prone paddling

\section{Introduction}

Lifesaving activities are usually associated with saving or protecting the lives of people in aquatic environments (Surf Life Saving Australia, 2016). Lifesaving is also a competitive sport that can help in developing the skills, knowledge, and techniques of lifesavers (International Life Saving Federation, 2015; Surf Life Saving Australia, 2011). When someone is drowning, lifesavers need to choose the most effective and quickest rescue method for reaching the victims. Among the various rescue methods, the rescue board is a fast and reliable means of reaching drowning people, and its use has become widespread at beaches around the world (Surf Life Saving Australia, 2016). In addition, most of the reported rescues were performed within $50 \mathrm{~m}$ off the shore (Gulbin et al., 1996; Japan Lifesaving Association, 2003-2010; Reilly et al., 2006). Taken together, the ability to achieve high board velocity (BV) within a short distance is a key competency for lifesavers involved in surf rescue activities.

Generally, lifesavers select the knee or prone 


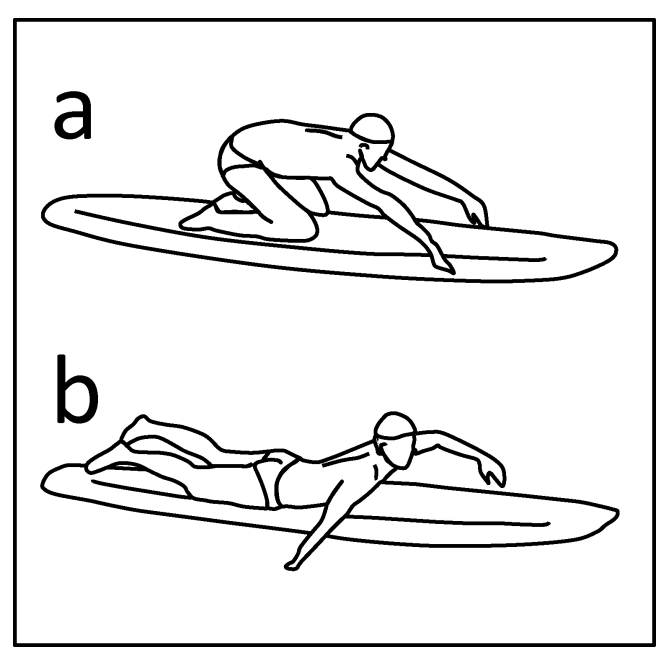

Figure 1 Two board paddling techniques, a: knee paddling technique (K-paddling); b: prone paddling technique (P-paddling).

paddling techniques depending on the surf conditions and their own paddling performance level (Figure 1). The knee paddling technique (Kpaddling) involves paddling in the kneeling position, with arm motions similar to the butterfly stroke of competitive swimming. The prone paddling technique (P-paddling) involves paddling in the prone position, with arm motions similar to the crawl stroke of competitive swimming (Surf Life Saving Australia, 2011, 2016). The key points of coaching for these two paddling techniques need to be looked at separately, and a correct paddling technique is essential to maximise BV (Surf Life Saving Australia, 2011). However, it is still unclear how top-level board paddlers produce a high BV using both paddling techniques.

Previous studies on competitive swimming (Toussaint and Beek, 1992), kayak (McDonnell et al., 2013), and stand-up paddle boarding (Schram et al., 2016) described the characteristics of swimming or boat velocity, which is represented as a product of stroke rate (SR) and stroke length (SL). Moreover, by analysing SR and SL in competitive swimming, differences in sex (Seifert et al., 2007), swimming events (Kennedy et al., 1990), race distance (Arellano et al., 1994; Craig and Pendergast, 1979), and skill levels (Chollet et al., 1997; Seifert et al., 2007) have been reported. Thus, stroke characteristic analysis in board paddling should also provide insight into how top-level board paddlers achieve a high BV.
There are a few studies focusing on lifesavers' board paddling. For example, in 40-m maximal board paddling among elite Japanese board paddlers, Miyama et al. (2013) reported that the peak $\mathrm{BV}$ was higher in K-paddling than in P-paddling, males achieved higher BV than females in both paddling techniques, and BV strongly correlated with SL for both paddling techniques. Gulbin et al. (1996) performed a comparison of a time trial for a 90-m slalom course of board paddling and reported that elite ironman athletes and full-time lifeguards achieved $22.7 \%$ and $20.4 \%$ faster times, respectively, than volunteer surf lifesavers. However, these studies did not clarify the differences in stroke characteristics based on the different performance levels of lifesavers. This means that it is still unknown how stroke characteristics differ in board paddling depending on the performance level. Such a comparison may provide useful information for lifesavers as well as coaches in improving BV. Therefore, we examined differences in the BV and stroke parameters (SR and SL) during 40-m maximal board paddling between elite and sub-elite board paddlers.

\section{Method}

\subsection{Participants}

This study was approved by the Institutional Ethics Human Research Committee of Osaka University of Health and Sport Sciences (number 11-19 and 12-28). Sixteen Japanese male lifesavers participated in this study after written informed consent was obtained. All participants had basic lifesaver qualifications accredited by the Japan Lifesaving Association. This qualification guaranteed that participants had acquired basic surf rescue skills including board paddling. There were two groups of participants, the elite group $(n=8)$ and sub-elite group $(n=8)$. The elite group comprised of finalists in the Board Race events held at the Japanese National Life Saving Championships. The Board Race events test a lifesaver's paddling abilities over a $600-\mathrm{m}$ distance off the shore in a typical surf setting, and their results are comprehensive indicators of paddlers' performance levels for various paddling skills, such as paddler's sprint, endurance, and surf negotiation. Two participants from the elite group were members of the Japanese national team and 
competed for the World Lifesaving Championships held by the International Life Saving Federation. The sub-elite group consisted of lifesavers who did not participate in the Board Race events or did not qualify as finalists. The mean $( \pm \mathrm{SD})$ age, height, weight, body fat, and arm span were $25.6 \pm 3.5$ years, $1.72 \pm 0.06 \mathrm{~m}, 67.0 \pm 6.9 \mathrm{~kg}, 11.4 \pm 2.4 \%$, and $1.79 \pm 0.05 \mathrm{~m}$, respectively, for the elite group and $20.0 \pm 1.1$ years, $1.71 \pm 0.08 \mathrm{~m}, 64.8 \pm 7.2 \mathrm{~kg}$, $10.9 \pm 2.2 \%$, and $1.72 \pm 0.06 \mathrm{~m}$, respectively, for the sub-elite group.

\subsection{Experimental protocol}

All participants performed 40-m maximal board paddling using the $\mathrm{K}$ - and $\mathrm{P}$-paddling techniques on a straight course in a 50-m indoor pool. Each paddling performance was executed twice, with more than $3 \mathrm{~min}$ of rest between each trial. All participants were instructed to wear swimsuits for competitive swimming. Prior to the experimental trials, the participants performed a self-selected warm-up for $15 \mathrm{~min}$ in the pool and practised manoeuvres to stop the board safely short of the pool edge. The Kand P-paddling trials were performed in a counterbalanced order. In the starting position, participants were positioned on the board, with the board tail set at the wall of the pool edge. The start cue for the trial was a verbal call "Ready, go!" shouted out by the investigator. All participants performed the 40- $\mathrm{m}$ maximal board paddling and slowed down in the 40- to 45-m zone. A standard racing board (Dolphin Surf Craft, Australia; length, $3.17 \mathrm{~m}$; maximum width, $0.45 \mathrm{~m}$; weight, $7.9 \mathrm{~kg}$ ) conforming to the Equipment Standards of the International Life
Saving Federation (International Life Saving Federation, 2015) was used.

Three video cameras (Sony Handycam HDRXR350V, $30 \mathrm{fps}$, Japan) were installed on the poolside at a distance of $20 \mathrm{~m}$ from the trial course. The board and paddling motions were recorded from the right side of the pool to cover the trial from the 5- to 40-m distance (Figure 2). We divided the 40-m trial course into $5-\mathrm{m}$ zones and calculated the time when the nose of the board passed each zone to analyse the BV and stroke parameters. The first 5-m zone was excluded as it is impossible for the board nose to be set at $0 \mathrm{~m}$ (the pool wall). A stroke cycle began when the right hand entered the water and ended when that hand recovered and entered the water again. This stroke cycle can be divided into the propulsive phase and recovery phase (Draper and Sayers, 1986). The propulsive phase corresponded to the time from the hand's entry into the water to its release from the water. The recovery phase corresponded to the time from the hand's release from the water to its subsequent entry into the water. The average time taken for one stroke cycle (ST), the duration of the propulsive phase, and the duration of the recovery phase were computed for each zone, with both paddling techniques, respectively. ST, the duration of the propulsive phase, and the duration of the recovery phase were averaged for two or more stroke cycles in each zone. When two or more stroke cycles could not be recorded, a single stroke cycle was evaluated. BV $(\mathrm{m} / \mathrm{s})$ was calculated by dividing the $5-\mathrm{m}$ distance by the time required. SR was determined as the estimated number of strokes per minute based on ST, as follows: SR (strokes $/ \mathrm{min})=60 \times 1 / \mathrm{ST}$

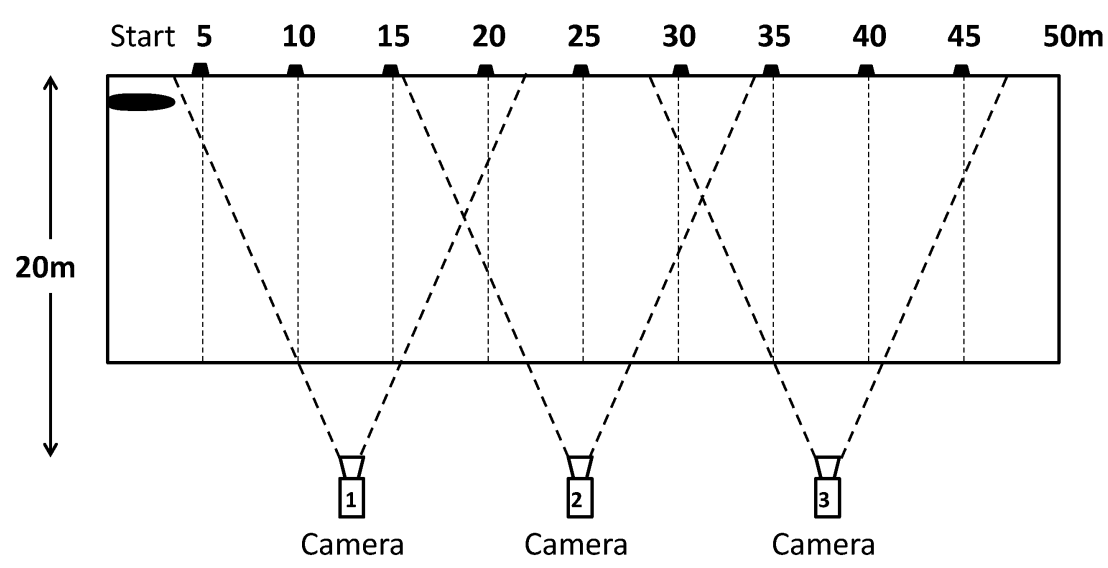

Figure 2 Experimental set-up of the 50-m indoor pool. 
(strokes/s). SL was determined as the distance in meters per stroke, as follows: SL $(\mathrm{m} /$ stroke $)=\mathrm{BV}$ $(\mathrm{m} / \mathrm{s}) \times \mathrm{ST}(\mathrm{s} / \mathrm{stroke})$. The relative duration of the propulsive and recovery phases were expressed as a percentage of ST. All variables were averaged over the two trials. All variables in the first zone were set as zero.

\subsection{Statistical analyses}

All statistical tests were performed using SPSS version 24 software program (IBM SPSS Statistics, Inc. ver. 24, Japan). The results were expressed as means \pm standard deviation (SD). Participants' body characteristics were compared using independent-samples $t$-test. The effect size of the independent-samples $t$-test was estimated using Cohen's $d$ (Cohen, 1988). A two-way repeated analysis of variance (ANOVA) was used to test the differences in BV, stroke parameters (SR, SL), and stroke phase parameters between the groups and the zones (two groups and eight zones). Mauchly's test of sphericity was used to assess data variance, and a Greenhouse-Geisser correction was applied in cases where there was an assumption violation. Bonferroni correction was used for multiple comparisons. The effect size of the two-way ANOVA was estimated using partial eta squared $\left(\eta p^{2}\right)$ for analysis of variance (Ferguson, 2009). The Pearson correlation coefficient was determined to test the relationship between $\mathrm{BV}$ and stroke parameters (SR, SL). Statistical significance was set at $P<0.05$.

\section{Results}

\subsection{Participants' body characteristics}

There were no significant differences in the height, weight, and body fat of the participants in the elite and sub-elite groups. However, there were significant differences between the two groups with regard to age $(t[8.3]=4.30, P<0.002, d=2.16)$ and arm span $(t[14]=2.5, P=0.02, d=1.27)$.

\subsection{Stroke parameters in K-paddling}

The elite group achieved peak BV over a shorter distance compared to the sub-elite group. The peak $\mathrm{BV}$ was $2.99 \pm 0.19 \mathrm{~m} / \mathrm{s}$ in the $25-$ to $30-\mathrm{m}$ zone for the elite group and $2.52 \pm 0.17 \mathrm{~m} / \mathrm{s}$ in the 30 - to
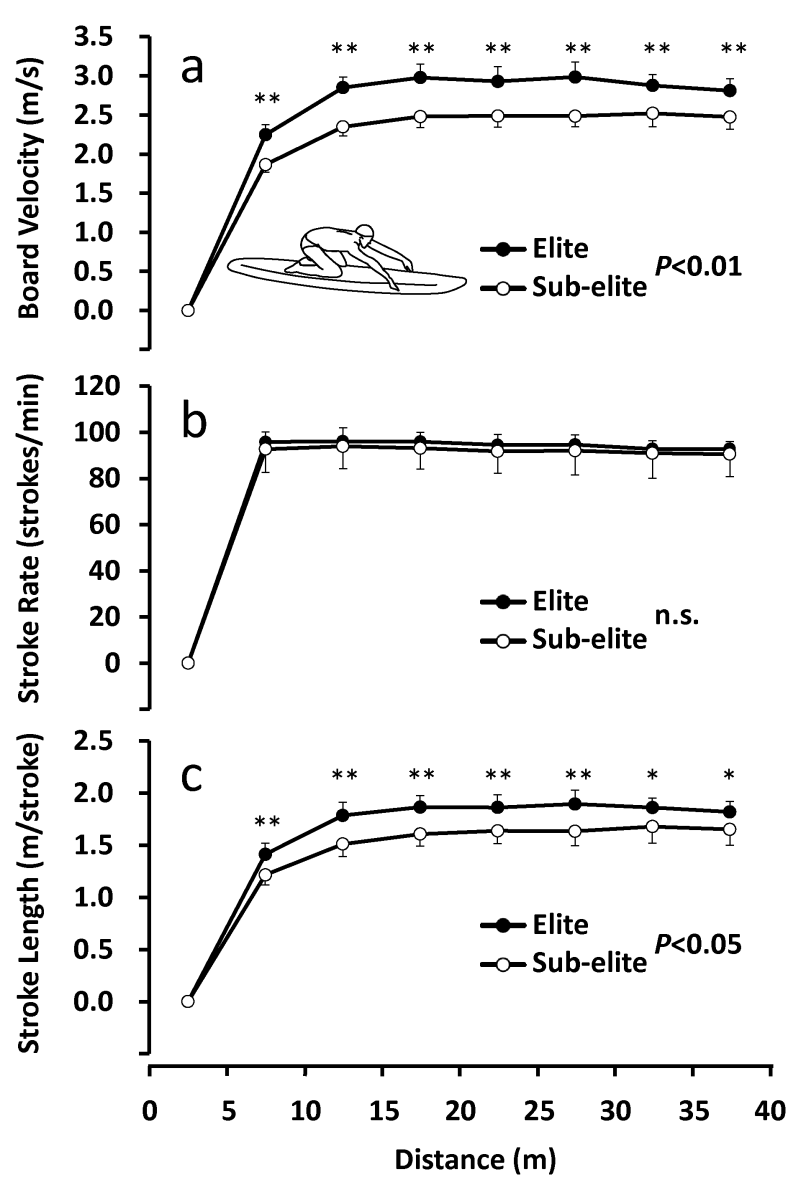

Figure 3 The changes in the board velocity (a), stroke rate (b), and stroke length (c) for knee paddling in the elite group (O) and sub-elite group $(\bigcirc)$. The mean $( \pm \mathrm{SD})$ values of eight participants are shown. $* *$ : a significant $(P<0.01)$ difference between the two groups. $*$ : a significant $(P<0.05)$ difference between the two groups.

35-m zone for the sub-elite group (Figure 3-a). There was a significant interaction effect for BV between the elite and sub-elite groups $(F[7,98]=$ 14.47, $\left.P<0.001, \eta p^{2}=0.51\right)$. In addition, a significant group effect was also found between the two groups $\left(F[1,14]=44.23, P<0.001, \eta p^{2}=0.76\right)$. Multiple comparisons revealed that the $\mathrm{BV}$ values of the elite group were significantly higher $(P<0.01)$ than those of the sub-elite group for all zones (Figure 3-a).

The SR values at the peak BV zone for the elite and sub-elite groups were $94.5 \pm 4.6$ strokes $/ \mathrm{min}$ and $90.9 \pm 10.8$ strokes/min, respectively. No significant interactions or group effects were found for the SR between the two groups (Figures 3-b).

The SL values at the peak BV zone for the elite and sub-elite groups were $1.89 \pm 0.13 \mathrm{~m} /$ stroke and $1.68 \pm 0.16 \mathrm{~m} /$ stroke, respectively. There were 
significant interaction $(F[3.7,51.8]=6.72, \quad P<$ $\left.0.001, \eta p^{2}=0.32\right)$ and group effects $(F[1,14]=$ 16.68, $P=0.001, \eta p^{2}=0.54$ ) observed for SL between the two groups. Multiple comparisons revealed that the SL values of the elite group were significantly $(P<0.05)$ higher than those of the subelite group for all zones (Figure 3-c).

The relative duration of the propulsive and recovery phases at the peak BV zone was $58.5 \pm 5.7 \%$ and $41.5 \pm 5.7 \%$ for the elite group and $57.9 \pm 4.8 \%$ and $42.1 \pm 4.8 \%$ for the sub-elite group, respectively. No significant interactions or group effects were found for the relative duration of the propulsive and recovery phases between the two groups.

\subsection{Stroke parameters in P-paddling}

The elite group achieved peak BV over a shorter distance than the sub-elite group. The peak BV was $2.77 \pm 0.21 \mathrm{~m} / \mathrm{s}$ in the 15 - to 20 - $\mathrm{m}$ zone for the elite group and $2.46 \pm 0.17 \mathrm{~m} / \mathrm{s}$ in the 20 - to 25 -m zone for the sub-elite group (Figure 4-a). There was a significant interaction effect for $\mathrm{BV}$ between the elite and sub-elite groups $(F[2.3,31.9]=6.26, P=0.004$, $\eta p^{2}=0.31$ ). In addition, a significant group effect was found between the two groups $(F[1,14]=8.92$, $P=0.01, \eta p^{2}=0.39$ ). Multiple comparisons revealed that the BV values of the elite group were significantly higher $(P<0.05)$ than those of the subelite group from the 5- to $10-\mathrm{m}$ zone to the 30 - to 35-m zone (Figure 4-a).

The SR values at the peak BV zone for the elite and sub-elite groups were $90.5 \pm 11.5$ strokes $/ \mathrm{min}$ and $83.5 \pm 7.7$ strokes/min, respectively. A significant interaction effect $(F[1.8,25.8]=3.73, P=$ $0.04, \eta p^{2}=0.21$ ) was observed for the SR between the two groups. However, the group effect between their performance levels was not significant. Multiple comparisons revealed that the SR values of the elite group were significantly $(P=0.03)$ higher than those of the sub-elite group in the 5 - to $10-\mathrm{m}$ zone (Figure 4-b).

The SL values at the peak BV zone for the elite and sub-elite groups were $1.87 \pm 0.33 \mathrm{~m} /$ stroke and $1.77 \pm 0.08 \mathrm{~m} /$ stroke, respectively. There were no significant interactions or group effects found for SL between the two groups (Figure 4-c).

The relative duration of the propulsive and recovery phases at peak BV was $59.1 \pm 2.1 \%$ and $40.9 \pm$ $2.1 \%$ for the elite group and $59.0 \pm 2.6 \%$ and 41.0

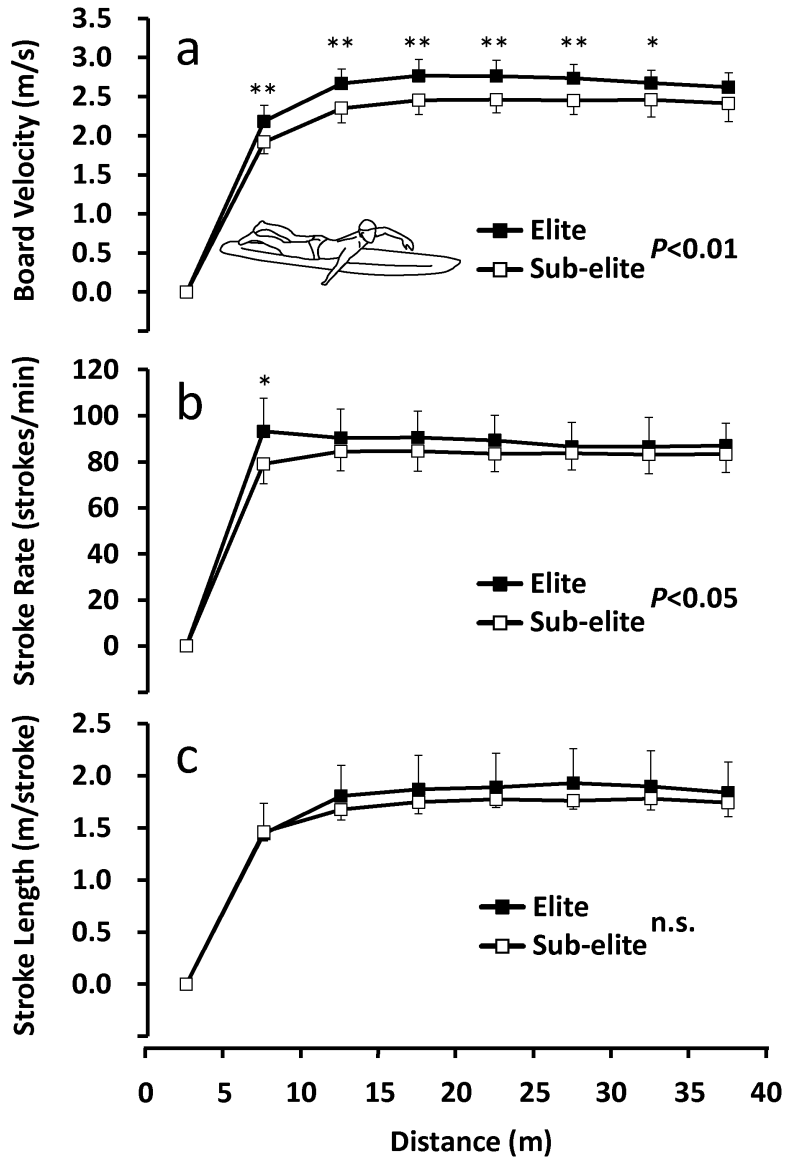

Figure 4 The changes in the board velocity (a), stroke rate (b), and stroke length (c) for prone paddling in the elite group ( $\square$ ) and sub-elite group $(\square)$. The mean $( \pm$ SD) values of eight participants are shown. $* *$ : a significant $(P<0.01)$ difference between the two groups. $*$ : a significant $(P<0.05)$ difference between the two groups.

$\pm 2.6 \%$ for the sub-elite group, respectively. No significant interactions or group effects were found for the relative duration of the propulsive and recovery phases between the two groups.

\subsection{Correlation between BV and stroke parameters}

In K-paddling, a significant correlation was found between the BV and SL in the peak BV zone $(r=$ $0.78, P=0.02)$ in the elite group. There was no correlation identified between the $\mathrm{BV}$ and stroke parameters in the peak BV zone in the sub-elite group (Table 1). In P-paddling, a significant correlation in the peak BV zone was found between the BV and SL $(r=0.77, P=0.03)$ in the elite group and between the BV and SR in the sub-elite group $(r=$ $0.88, P=0.004)$ (Table 1). 
Table 1 The correlation coefficients between the peak board velocity and stroke parameters for knee and prone paddling in the elite group and sub-elite group.

\begin{tabular}{cccc}
\hline & & $\begin{array}{c}\text { SR } \\
(\text { stroke/min) }\end{array}$ & $\begin{array}{c}\text { SL } \\
(\mathrm{m} / \text { stroke) }\end{array}$ \\
\hline K-paddling & & & \\
Elite & Peak BV (m/s) & 0.26 & $0.78^{*}$ \\
Sub-elite & Peak BV (m/s) & 0.56 & -0.03 \\
\hline P-paddling & & & \\
Elite & Peak BV (m/s) & -0.56 & $0.77^{*}$ \\
Sub-elite & Peak BV (m/s) & $0.88^{*}$ & -0.31 \\
\hline
\end{tabular}

* significant correlation between Peak BV and stroke parameters $(P<0.05)$.

BV: board velocity; SR: stroke rate; SL: stroke length

\section{Discussion}

This study examined differences in BV and stroke parameters (SR, SL) during 40-m maximal knee and prone paddling between elite and sub-elite paddlers. The results demonstrated that BVs were significantly higher in the elite group than in the sub-elite group, for both paddling techniques. Furthermore, the differences in the BVs between the two groups were due to the significantly greater SL in Kpaddling and the significantly higher $\mathrm{SR}$ in $\mathrm{P}$ paddling, respectively, in the elite group. In addition, the peak BVs were significantly related to the SL values of the elite group, for both paddling techniques.

\subsection{Differences in the peak $B V$ in $K$ - and $P$ - paddling}

Compared with the sub-elite group, the peak BV in the elite group was $18.7 \%$ higher with K-paddling (elite, $2.99 \mathrm{~m} / \mathrm{s}$ vs. sub-elite, $2.52 \mathrm{~m} / \mathrm{s}$ ) and $12.6 \%$ higher with P-paddling (elite, $2.77 \mathrm{~m} / \mathrm{s}$ vs. sub-elite, $2.46 \mathrm{~m} / \mathrm{s}$ ). Moreover, the elite group needed $30 \mathrm{~m}$, compared to the $35 \mathrm{~m}$ needed by the sub-elite group, to reach peak BV with K-paddling. The elite group needed $20 \mathrm{~m}$, compared to the $25 \mathrm{~m}$ needed by the sub-elite group, to reach peak BV with Ppaddling (Figures 3-a and 4-a). These findings indicate that the elite group demonstrated superior acceleration to achieve higher BVs in shorter distances with both paddling techniques. In an actual surf rescue situation, the ability to accelerate is an important factor in reaching a drowning person quickly within a short period time. Our study results suggest that assessing board paddlers over a $35-\mathrm{m}$ course can be effective for evaluating the peak BV with both paddling techniques.

\subsection{Differences in the stroke parameters with $\mathrm{K}$ - and P-paddling}

\subsubsection{K-paddling}

For K-paddling, the elite group demonstrated a higher BV and a longer SL than the sub-elite group throughout the course of the $40-\mathrm{m}$ board paddling, although SR was similar between the two groups (Figure 3). These findings suggest that the difference in the BVs between the two groups was due mainly to the higher SL values of the elite group. Our study results corroborate with those of competitive swimming, confirming an association between greater SL and a higher performance level in events with 50- to 200-m distance (Arellano et al., 1994; Chollet et al., 1997; Kennedy et al., 1990; Seifert et al., 2007; Toussaint and Beek, 1992). Moreover, a significant positive correlation $(r=0.78)$ between $\mathrm{BV}$ and SL was found only in the elite group for K-paddling (Table 1). Previous studies have described the positive correlation between SL and BV in K-paddling during simulated racing (Draper and Sayers, 1986) and during maximal board paddling (Miyama et al., 2013). Our study results corroborate these observations and also demonstrate a new finding that SL played a greater role in achieving peak BV than SR, only in the elite group and not in the sub-elite group. Taken together, SL is considered to be a useful indicator for both distinguishing performance levels and achieving higher BV in K-paddling.

The differences in the SL between individuals of different performance levels are explained by several possible reasons. First, the greater arm span of the elite group may have contributed to their greater SL and BV. In our study, the arm span of the elite group was significantly greater than that of the subelite group. In accordance with the finding of competitive swimming with front crawl technique (Seifert et al., 2007), a longer arm span is known to result in greater SL and contribute to higher BV. Second, Draper and Sayers (1986) reported that in male paddlers, the slowest BV paddler extended the shoulder joint 10 degrees more than the fastest BV paddler at the end of propulsive phase in $\mathrm{K}$ paddling. They also reported that there was a strong 
positive correlation between BV and SL $(r=0.95)$, suggesting that greater extension of the shoulder joint in the slowest $\mathrm{BV}$ paddler did not effectively contribute towards extending the SL. In the present study, for K-paddling, the elite group's SL at the peak BV zone was $12.5 \%$ higher, but the relative duration of the propulsive phase was only $1 \%$ longer than the sub-elite group. Therefore, there may be differences between the two groups in terms of their hands and arms' motions to produce greater horizontal forces in the propulsive phase with similar relative duration of stroke phases. Further biomechanical studies in hand and arm motions in the propulsive phase are needed to confirm the factors contributing to the elite paddlers having a greater SL in K-paddling.

\subsubsection{P-paddling}

With P-paddling, the elite group demonstrated a higher BV and SR than the sub-elite group throughout the course of the 40-m board paddling, although SL was similar between the two groups (Figure 4). Our study results identified that the elite group paddled faster and stronger, but with similar SL values as the sub-elite group, in the 5- to $10-\mathrm{m}$ zone. Pelayo et al. (1996) described that increasing SR was the most important means of increasing freestyle swimming velocity in short-distance competitions $(50 \mathrm{~m}, 100 \mathrm{~m}$, and $200 \mathrm{~m})$. Therefore, it is possible that the elite paddlers increased their SR values to accelerate to a greater $\mathrm{BV}$ in the 5 - to $10-\mathrm{m}$ zone. Grimston and Hay (1986) indicated that the ST can be governed by the range of motion at the shoulder joint and the average angular velocity of the upper extremity over range in competitive swimming. Considering this with the board paddling movement, the stroking pattern and the power output of the stroke movement influence SR. In the present study, there were no significant group differences in both the relative duration of the propulsive and recovery phases, respectively. Therefore, the efficient stroke pattern over a complete paddling stroke phase in the elite group may have contributed to their higher SR. Furthermore, in competitive surfing, Mendez-Villanueva and Bishop (2005) reported that higher levels of upper-body strength and power assist surfers to generate rapid and explosive arm strokes in P-paddling, which is similar to a technique in lifesaving. Therefore, there is a pos- sibility that the higher power output of the upperbody in the elite group contributed to their higher SR.

Despite the arm span differences between the elite and sub-elite groups, SL did not significantly differ between the two groups in P-paddling. Larger SD values of SL in P-paddling were observed than those of K-paddling for the elite group (Figures 3 and 4). These results may have caused no differences in SL between the two groups and indicated high individual variability in stroke characteristics (e.g. SL type or SR type) for the elite group in the Ppaddling. However, SL showed a significant positive correlation $(r=0.77)$ with $\mathrm{BV}$ in the elite group for P-paddling. In contrast, SR was the only parameter that showed a significant positive correlation $(r=0.88)$ with $\mathrm{BV}$ in the sub-elite group for $\mathrm{P}$ paddling (Table 1). Similar to K-paddling, SL is considered to be a useful indicator for achieving higher BV in P-paddling.

The differences in the stroke parameters between the elite and sub-elite groups in P-paddling appeared to be a reversal of the relationship observed in K-paddling. This may be explained by the differences in the degree of difficulty of acquiring the two paddling skills. K-paddling requires more skill as it is more difficult to maintain balance in the kneeling position (Surf Life Saving Australia, 2016). Draper and Sayers (1986) reported that non-expert paddlers relied heavily on the use of arms in both paddling positions and failed to fully utilise all possible body segments during K-paddling. It is reasonable to assume that there are different models of progress in skill acquisition for the two paddling techniques. This is because, in the course of training for Kpaddling, the sub-elite paddlers achieved a similar SR as did elite paddlers, before their SL improved. In contrast, with P-paddling, sub-elite paddlers achieved a similar SL as did elite paddlers before their SR improved.

\subsubsection{Practical applications}

Our results have several practical applications. First, coaches may assess peak BV by measuring the time required for completing a $35-\mathrm{m}$ accelerating board paddling course. Second, board paddlers and coaches should understand the differences in the SR-SL ratios for different performance levels based on the results of the present study. They should 
focus on the increases in the SL and SR values for $\mathrm{K}$-paddling and P-paddling, respectively, based on the SR-SL ratios of the elite group in the present study, when improving sub-elite paddlers' BV to reach elite paddler levels. Third, similar to the training methods used for competitive swimming (Toussaint and Beek, 1992), lifesavers' training should aim at reducing the sum of the required time for completion and the number of strokes for $35-\mathrm{m}$ maximal board paddling, as a means of improving paddlers' SL.

Several potential limitations to the present study should be noted. First, we observed only eight board paddlers in each group to identify the differences between the different group levels. It was difficult to recruit top-level board paddlers as participants in this study because we defined the criterion for the elite group as the finalists of the Board Race event in the highest-level championships in Japan, and the number of the finalists is limited. Second, only male board paddlers were compared in terms of stroke characteristics in the present study. Thus, female board paddlers should also be compared among the similar groups to clarify sex differences.

\section{Conclusion}

The present study identified that the differences in the BVs during 40-m maximal board paddling between elite and sub-elite paddlers were due to a greater SL in K-paddling and a higher SR in Ppaddling, respectively, in the elite group. Furthermore, our study concluded that SL may be a useful indicator during training to improve peak BV.

\section{Acknowledgments}

This work was supported by JSPS KAKENHI Grant Number 24500752.

\section{References}

Arellano, R., Brown, P., Cappaert, J., and Nelson, R. C. (1994). Analysis of 50-, 100-, and 200-m freestyle swimmers at the 1992 Olympic Games. J. Appl. Biomech., 10: 189-199.

Chollet, D., Pelayo, P., Delaplace, C., Tourny, C., and Sidney, M. (1997). Stroking characteristic variations in the 100-M freestyle for male swimmers of differing skill. Percept. Mot. Skills, 85: 167-177.

Cohen, J. (1988). Statistical power analysis for the behavioral sciences (2nd ed.). Hillsdale: Lawrence Erlbaum Associates.

Craig, A. B. and Pendergast, D. R. (1979). Relationships of stroke rate, distance per stroke, and velocity in competitive swimming. Med. Sci. Sports, 11, 278-283.
Draper, J. A. and Sayers, M. G. L. (1986). Biomechanical analysis of surf board paddling. Australian Sports Commission. National Sports Research Centre.

Ferguson, C. J. (2009). An Effect Size Primer: A Guide for Clinicians and Researchers. Prof. Psychol. Res. Pr., 40, 532-538.

Grimston, S. K. and Hay, J. G. (1986). Relationships among anthropometric and stroking characteristics of college swimmers. Med. Sci. Sports Exerc., 18, 60-68.

Gulbin, J. P., Fell, J. W., and Gaffney, P. T. (1996). A physiological profile of elite surf ironmen, full time lifeguards \& patrolling surf life savers. Aust. J. Sci. Med. Sport, 28, 86-90.

International Life Saving Federation. (2015). ILS Competition Rule Book (2015-2019 ed.). Leuven: International Life Saving Federation.

Japan Lifesaving Association. (2003-2010). Patrol toukei houkokusho [Patrol Log Report]. http://jla.gr.jp/accident_ prevention/rescue_patrol/patrol_statistics.html (accessed 2018-04-21). (in Japanese).

Kennedy, P., Brown, P., Chengalur, S. N., and Nelson, R. C. (1990). Analysis of male and female Olympic swimmers in the 100-meter events. Int. J. Sport Biomech., 6, 187-197.

McDonnell, L. K., Hume, P. A., and Nolte, V. (2013). A deterministic model based on evidence for the associations between kinematic variables and sprint kayak performance. Sports Biomech., 12, 205-220.

Mendez-Villanueva, A. and Bishop, D. (2005). Physiological aspects of surfboard riding performance. Sports Med., 35, 55-70.

Miyama, M., Uematsu, A., Endo, H., Arai, H., Nakatsuka, K., and Araki, M. (2013). Kinematic characteristics during maximal board paddling: Comparison between two board paddling techniques on gender. Jpn. J. Mari. Activity, 2, 1-8. (in Japanese).

Pelayo, P., Sidney, M., Kherif, T., Chollet, D., and Tourny, C. (1996). Stroking characteristics in freestyle swimming and relationships with anthoropometric characteristics. J. Appl. Biomech., 12, 197-206.

Reilly, T., Wooler, A., and Tipton, M. (2006). Occupational fitness standards for beach lifeguards. Phase 1: the physiological demands of beach lifeguarding. Occup. Med., 56, 6-11.

Schram, B., Hing, W., and Climstein, M. (2016). Profiling the sport of stand-up paddle boarding. J. Sports Sci., 34, 937-944.

Seifert, L., Chollet, D., and Chatard, J. C. (2007). Kinematic changes during a 100-m front crawl: effects of performance level and gender. Med. Sci. Sports Exerc., 39, 1784-1793.

Surf Life Saving Australia. (2011). Board paddling. Surf Life Saving Australia Coaching Manual (5th ed.). NSW: Surf Life Saving Australia.

Surf Life Saving Australia. (2016). Public safety and aquatic rescue (34th ed.). NSW: Elsevier Australia.

Toussaint, H. M. and Beek, P. J. (1992). Biomechanics of competitive front crawl swimming. Sports Med., 13, 8-24. 
Name:

Motoyoshi Miyama

\section{Affiliation:}

Faculty of Management and Information Sciences, Josai International University

Address:

1 Gumyo, Togane, Chiba, 283-8555, Japan

Brief Biographical History:

2004-Present Josai International University, Chiba, Japan 2007 Ph.D. in Medicine, Showa University, Tokyo, Japan 1996 Master of Sports Science, The University of New South Wales, Sydney, Australia

1992 Master of Physical Education, Nippon Sport Science University, Tokyo, Japan

\section{Main Works:}

-Miyama, M., Uematsu, A., Endo, H., Arai, H., Nakatsuka, K., and Araki, M. (2013). Kinematic characteristics during maximal board paddling: Comparison between two board paddling techniques on gender. Jpn. J. Mari. Activity, 2, 1-8. (in Japanese).

- Miyama, M. and Nosaka, K. (2007). Protection against muscle damage following 50 drop jumps conferred by 10 drop jumps. J. Strength Cond. Res. 21(4), 1087-1092.

-Miyama, M. and Nosaka, K. (2004). Muscle damage and soreness following repeated bouts of consecutive drop jumps. Adv. Exer. Sport Physiol., 10(3), 63-69.

- Miyama, M. and Nosaka, K. (2004). Influence of surface on muscle damage and soreness induced by consecutive drop jumps. J. Strength Cond. Res. 18(2), 206-211.

Membership in Learned Societies:

- Japan Society of Physical Education, Health and Sport Sciences

- Japanese Society of Physical Fitness and Sports Medicine - Japanese Society of Sport Psychology 